\title{
Influence of the structural state on mechanical properties of tin babbit under upsetting
}

\author{
I. Sh. Valeev, A. Kh. Valeeva ${ }^{\dagger}$, A. Kh. Akhunova \\ †valeevs@mail.ru \\ Institute for Metals Superplasticity Problems RAS, 39 Khalturin St., 450001, Ufa, Russia
}

\begin{abstract}
The behavior of a material in extreme regimes of operation in large constructions can be calculated with the use of modern software codes (ANSYS, DEFORM, LS-Dyna). However, they do not have a sufficient basis for the properties of metals and alloys. Therefore, it is necessary to input experimental mechanical properties of a studied material for an adequate description of a process. The present work aims to obtain the mechanical properties of babbit $\mathrm{Sn} 11 \% \mathrm{Sb} 5,5 \% \mathrm{Cu}$ in different structural states for a use in computer modeling in the software product Deform. As an object of the study, babbit of a chemical composition $\mathrm{Cu}$ 5.5-6.5 wt.\%, Sb 10-12 wt.\%, Sn - rest was chosen. Two different structural states of the alloy were obtained at different crystallization rates: the first by casting with air cooling and the second by casting with cooling in running water (rapid cooling). The mechanical properties were determined by upsetting tests according to GOST $8817-82$ standart. An Axiovert-100A microscope with the KSLite image processing program was used for optical metallography. A finite element simulation of a large-sized sliding bearing during operation in a two-dimensional formulation was carried out using the DEFORM-2D software. To evaluate the degree of destruction of the bearing during operation a scalar parameter of damage was determined using the model of metal damage accumulation during monotonic deformation. It is shown that rapid cooling leads to the formation of a structure with small intermetallic particles uniformly distributed in the matrix phase. Such a structure is characterized by enhanced mechanical properties, and computer simulation allows predicting its high wear resistance in a large-sized sliding bearing during operation.
\end{abstract}

Keywords: babbit, mechanical properties, structure, computer modeling.

\section{Механические свойства баббита Б83 с различной структурой при осадке}

\author{
Валеев И.Ш., Валеева А. Х. ${ }^{\dagger}$, Ахунова А.Х. \\ Институт проблем сверхпластичности металлов РАН, ул. Ст. Халтурина 39, 450001, Уфа, Россия
}

Современные пакеты прикладных программ (ANSYS, Deform, LS-Dyna) позволяют рассчитать поведение материала при предельных или аварийных режимах работы для конструкций любого размера. Однако они не имеют достаточной базы по свойствам металлов и сплавов, поэтому для адекватного описания процесса необходимо вводить экспериментальные механические свойства исследуемого материала. Цель работы - получить механические свойства баббита Б83 с различным структурным состоянием, для использования при компьютерном моделировании в среде программного продукта DEFORM. В качестве объекта исследования выбран баббит Б83 химического состава $\mathrm{Cu}-5,5-6,5 \%$ вес., $\mathrm{Sb}-10-12 \%, \mathrm{Sn}-$ ост. с двумя различными структурными состояниями, полученными при разной скорости кристаллизации в результате литья в форму с охлаждением на воздухе и при литье в форму с охлаждением проточной водой (скоростное охлаждение). Механические свойства определяли при испытаниях на осадку согласно ГОСТ 8817 - 82. Оптическую металлографию проводили на микроскопе «Axiovert-100A», с программой обработки изображения KSLite. C помощью пакета прикладных программ DEFORM-2D было проведено компьютерное моделирование работы крупногабаритного подшипника скольжения в двумерной постановке. Для оценки степени разрушения подшипника определяли скалярный параметр поврежденности с использованием модели накопления поврежденности металла при монотонной деформации. Показано, что скоростное охлаждение приводит к формированию структуры с мелкими, раздробленными частицами интерметаллидных фаз, равномерно распределенными в матричной фазе. Такая структура характеризуется повышенными механическими свойствами, а компьютерное моделирование позволяет прогнозировать ее высокую стойкость при изнашивании вкладыша из баббита Б83 при работе крупногабаритного подшипника скольжения.

Ключевые слова: баббит, механические свойства, структура, компьютерное моделирование. 


\section{1. Введение}

Высокооловянистый баббит Б83 широко используется в качестве материала для вкладышей подшипников скольжения многих крупногабаритных агрегатов (например, турбин гидроэлектростанций) [1,2]. Структура Б83 состоит из трех фаз: $\alpha$-фазы, являющейся твердым раствором сурьмы и меди в олове, $\beta$-фазы, представляющей собой кристаллы SnSb с гексагональной и ромбоэдрической решеткой и мелкой $\eta$-фазы $\left(\mathrm{Cu}_{6} \mathrm{Sn}_{5}\right)$. Эксплуатационные свойства баббита в значительной степени определяются его структурой и механическими свойствами [2]. Известно, что измельчение крупных интерметаллидных частиц, приводит к повышению усталостной прочности и способствует увеличению ресурса работы подшипников скольжения [3-6]. В настоящее время существуют методы, позволяющие произвести расчетное обоснование прочностной надежности любой конструкции. С использованием компьютерного моделирования появляется возможность рассчитать поведение материала при предельных или аварийных режимах работы для конструкций любого размера. Известные пакеты прикладных программ (ANSYS, DEFORM, LS-Dyna) не имеют достаточной базы по свойствам металлов и сплавов, поэтому для адекватного описания процесса необходимо вводить экспериментальные механические свойства исследуемого материала. Однако в литературе недостаточно информации о влиянии структуры, в частности, размера $\beta$-фазы на механические свойства баббита Б83.

Цель работы - получить механические свойства баббита Б83 с различным структурным состоянием, для использования при компьютерном моделировании в среде программного продукта DEFORM-2D.

\section{2. Материалы и методики}

В качестве объекта исследования выбран баббит Б83 химического состава $(\mathrm{Cu}-5,5-6,5 \%$ вес., $\mathrm{Sb}-10-12 \%$, $\mathrm{Sn}-$ ост.) с двумя различными структурными состояниями, полученными при различной скорости кристаллизации, в результате литья в форму с охлаждением на воздухе и при литье в форму с охлаждением проточной водой (скоростное охлаждение). Обычное литье использовали, так как это стандартный промышленный способ получения баббитового вкладыша при заливке подшипника скольжения. Скоростное охлаждение при литье позволяет получить мелкие частицы $\beta$-фазы $[3,5,7]$, что обычно повышает триботехнические характеристики исследуемого сплава.

Испытания на осадку выполнялись согласно ГОСТ 8817-82. Образцы с квадратным поперечным сечением площадью 100 мм² $^{2}$ и высотой 15 мм подвергались деформации осадкой до степени деформации $65 \%$ при температуре $T=20^{\circ} \mathrm{C}$ и скоростях деформации $\dot{\varepsilon}=10^{-4}-10^{-2} \mathrm{c}^{-1}$ на универсальном динамометре фирмы «Instron». Осаживали по 3 образца на каждую скорость деформации, ошибка измерений составляла $\pm 3 \%$.

Для выявления структуры баббита образцы сначала подвергали механической полировке, затем - химиче- скому травлению раствором азотной и уксусной кислоты в глицерине [8]. Оптическую металлографию проводили на микроскопе «Axiovert-100A», с программой обработки изображения KSLite.

Компьютерное моделирование разрушения подшипника в результате давления вала при отсутствии масла (аварийные условия работы) проводили в двумерной постановке с помощью пакета прикладных программ DEFORM-2D. Подшипник состоял из трех элементов: корпуса из чугуна с геометрическими размерами $400 \times 200$ мм по осям $X$ и $Y$, соответственно; вкладыша из баббита Б83 в виде полукольца с внутренним диаметром 300 мм и толщиной 6 мм; а также вращающегося вала диаметром 300 мм.

Конечно-элементная модель подшипника приведена на рис. 1. Валу задавались свойства абсолютно жесткого тела, вкладыш и корпус принимались упруго-пластичными. В качестве материала корпуса был выбран сплав из стандартной библиотеки DEFORM-2D, близкий по свойствам к чугуну. Материал образцов описывался полученными экспериментальными кривыми «напряжение-деформация». Контактные условия на границе «валвкладыш» описывались моделью трения сдвига (shear). Величина коэффициента трения принималась равной 0.3 .

Деформируемые тела разбивали на двадцатиузловые изопараметрические конечные элементы с квадратичной аппроксимацией поля перемещений. Количество элементов вкладыша и корпуса - 4150 для каждого. Скорость вращения вала составляла 314 рад/с, время вращения -5 часов. Испытания проводились при комнатной температуре.

Для оценки степени разрушения подшипника скольжения при работе в результате давления крутящегося вала определяли скалярный параметр поврежденности с использованием предложенной В.Л. Колмогоровым формулы

$$
\Psi=\Sigma \Delta \Lambda / \Lambda_{p}[K],
$$

где $\Delta \Lambda$ - приращение степени деформации сдвига, $\Lambda_{p}-$ предельная степень деформации при данном коэффициенте жесткости напряжённого состояния [K] [9-10].

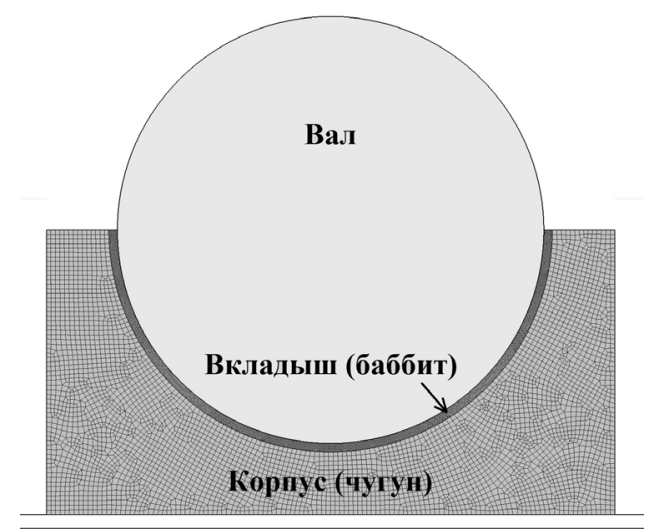

Рис. 1. Конечно-элементная модель подшипника, состоящего из вала, вкладыша и корпуса.

Fig. 1. Finite element model of the bearing consists a shaft, a bushing and the shell. 


\section{3. Результаты и обсуждение}

Микроструктура баббита Б83, полученного при различной скорости кристаллизации, представлена на рис. 2.

На фотографиях, полученных оптической металлографией, видно, что баббит в литом состоянии характеризуется микроструктурой с крупными кубическими частицами $\beta$-фазы SnSb размером 100-200 мкм и $\eta$-фазой в виде крупных звездочек - игл соединения $\mathrm{Cu}_{6} \mathrm{Sn}_{5}$, неравномерно распределенными в матричной $\alpha$-фазе (рис. 2a). Повышение скорости кристаллизации при охлаждении водой приводит к уменьшению размера $\beta$-фазы на порядок, кроме того иглы $\eta$-фазы не успевают вырасти, оставаясь мелкими глобулярными частицами, при этом обе интерметаллидные фазы равномерно распределены в матрице (рис. 2b).

На рис. 3 приведены кривые «напряжение-деформация», полученные при осадке образцов из баббита Б83 с различной скоростью деформации. Видно, что при низкой скорости деформации, характер кривых практически одинаков, в то время как ее повышение приводит к более значительному росту напряжений в случае баббита, полученного при литье со скоростным охлаждением.
Компьютерное моделирование, проведенное с в среде программного продукта DEFORM-2D, с использованием полученных кривых «напряжение-деформация», позволяет прогнозировать высокую стойкость подшипника с баббитовым вкладышем, полученным по технологии литья со скоростным охлаждением, т. к. скалярный параметр поврежденности, рассчитанный при решении задачи, оказался на порядок ниже в случае его деформирования при аварийной работе (рис. 4).

\section{4. Заключение}

1. При испытаниях на осадку образцов из баббита Б83 с разной структурой, образовавшейся в результате изменения скорости кристаллизации, получены кривые «напряжение - деформация», которые использованы для задания механических свойств материала при моделировании в среде программного продукта DEFORM 2D.

2. Компьютерное моделирование позволяет прогнозировать высокую стойкость при аварийной работе крупногабаритного подшипника скольжения со вкладышем из баббита Б83 со структурой с мелкими, раздробленными частицами интерметаллидных фаз, равномер-
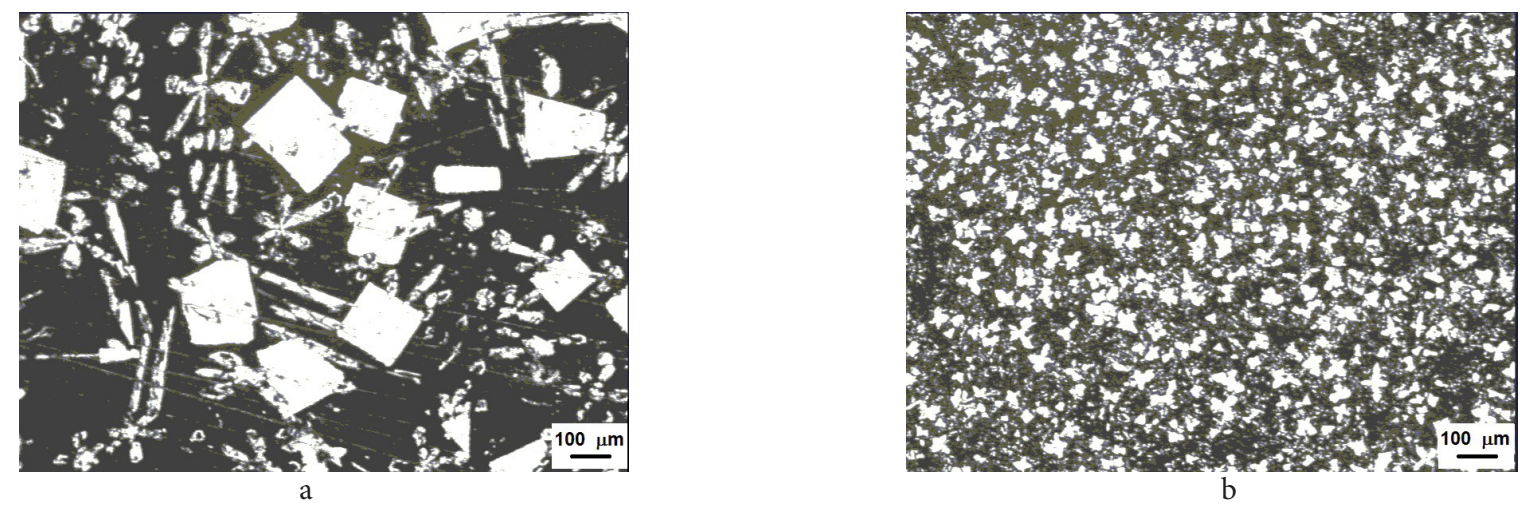

Рис. 2. Микроструктура баббита Б83: а) обычное литье, b) литье со скоростным охлаждением.

Fig. 2. Microstructure of babbit $\mathrm{Sn} 11 \% \mathrm{Sb} 5.55 \mathrm{Cu}$ : a) casting, b) casting with rapid cooling.

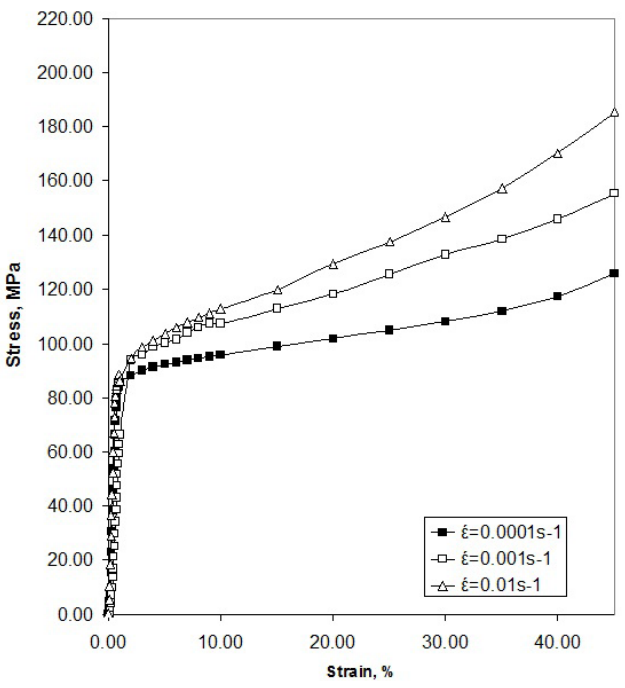

a

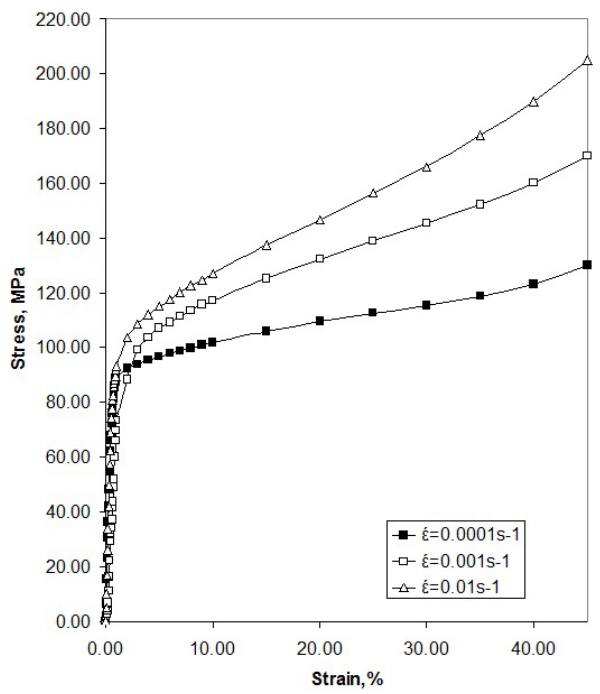

b

Рис. 3. Кривые «напряжение-деформация» баббита Б83 при различной скорости деформации: а) обычное литье, b) литье со скоростным охлаждением.

Fig. 3. Curves «stress — strain» of babbit $\mathrm{Sn} 11 \% \mathrm{Sb} 5.55 \mathrm{Cu}$ at different strain rate: a) casting, b) casting with rapid cooling. 


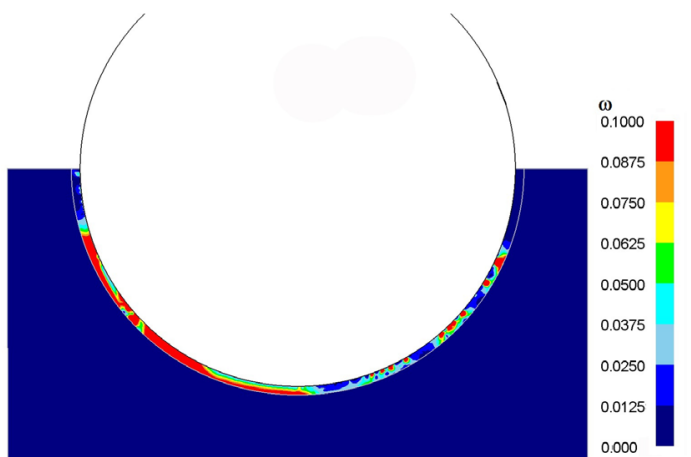

a

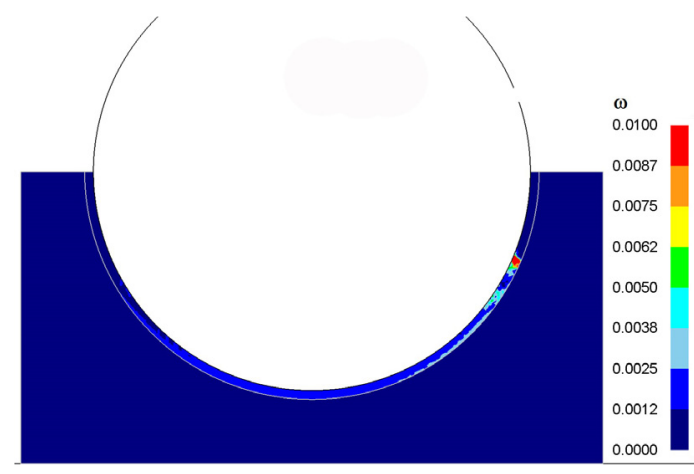

b

Рис. 4. Скалярный параметр поврежденности для подшипника со вкладышем из баббита Б83, полученным а) литьем, b) литьем со скоростным охлаждением.

Fig 4. Scalar parameter of damage for a bearing with a bushing out of babbit $\mathrm{Sn} 11 \mathrm{Sb} 5.5 \mathrm{Cu}$, obtained by a) casting, b) casting with rapid cooling.

но распределенными в матричной фазе, т. к. скалярный параметр поврежденности, рассчитанный при решении задачи, оказался на порядок ниже.

\section{Литература/Referencies}

1. A. I. Shpagin. Antifrictional alloys. M.: Metallurgya (1956) 326 p. (in Russian) [А.И. Шпагин. Антифрикционные сплавы. М.: Металлургия (1956) 326 c.]

2. Wear-resistant materials in chemical machine building. Handbook. Ed. Yu. M. Vinogradov. L.: Mashinostroyenie (1977) 256 p. (in Russian) [Износостойкие материалы в химическом машиностроении. Справочник. Под ред. Ю.М. Виноградова. Л.: Машиностроение (1977) 256 c.]

3. F. A. Sadykov, N. P. Barykin, I. Sh. Valeev, V. N. Danilenko. Journal of Materials Engineering and Performance. 12, $29-36$ (2003).

4. I.M. Lyubarskii, L.S. Palatnik. Metallofizika of friction. M.: Metallurgy (1976) 176 p. (in Russian) [И. М. Любарский, Л.С. Палатник. Металлофизика трения. М.: Металлургия (1976) 176 с.]

5. F. A. Sadykov, N.P. Barykin, I.Sh. Valeev. Strength of Materials 34, 196-199 (2002).

6. N.P. Barykin, R. F. Fazlyahmetov, A. Kh. Valeeva. Metal science and Heat Treatment. 48, 88-91 (2006).

7. A. Kh. Valeeva, I.Sh. Valeev, R. F. Fazlyakhmetov. Journal of Friction and Wear. 35. № 4, 311-315 (2014). DOI: $10.3103 /$ S1068366614040138

8. V.S. Kovalenko. Metallurgical reagents. M.: Metallurgya (1981) 120 p. (in Russian) [В.С. Коваленко. Металлографические реактивы. М.: Металлургия (1981) $120 \mathrm{c.}$ ]

9. V.L. Kolmogorov. Plasticity and destruction. M.: Metallurgy (1977) 336 p. (in Russian) [В. Л. Колмогоров Пластичность и разрушение. М.: Металлургия (1977) $336 \mathrm{c}$.

10. A.A. Bogatov. Mechanical properties and metals destruction methods. Ekaterinburg: Ural State Technical University (2002) 329 p. (In Russian) [А.А. Богатов. Механические свойства и методы разрушения металлов: Екатеринбург: ГОУ ВПО УГТУ - УПИ (2002) 329 c.] 